\title{
Modeling Particle Transport in Astrophysical Outflows and Simulations of Associated Emissions from Hadronic Microquasar Jets
}

\author{
D. A. Papadopoulos, ${ }^{1,2}$ O. T. Kosmas $\mathbb{D}^{3},{ }^{3}$ and S. Ganatsios ${ }^{2}$ \\ ${ }^{1}$ Department of Electrical and Computer Engineering, University of Western Macedonia, Kozani, Greece \\ ${ }^{2}$ Theoretical Physics Section, University of Ioannina, GR-45110 Ioannina, Greece \\ ${ }^{3}$ Modelling and Simulation Center, MACE, University of Manchester, Sackville Street, Manchester, UK \\ Correspondence should be addressed to O. T. Kosmas; odysseas.kosmas@manchester.ac.uk
}

Received 8 September 2021; Revised 17 December 2021; Accepted 28 December 2021; Published 8 February 2022

Academic Editor: Mariana Frank

\begin{abstract}
Copyright (c) 2022 D. A. Papadopoulos et al. This is an open access article distributed under the Creative Commons Attribution License, which permits unrestricted use, distribution, and reproduction in any medium, provided the original work is properly cited. The publication of this article was funded by $\mathrm{SCOAP}^{3}$.
\end{abstract}

\begin{abstract}
In this work, after improving the formulation of the model on particle transport within astrophysical plasma outflows and constructing the appropriate algorithms, we test the reliability and effectiveness of our method through numerical simulations on well-studied galactic microquasars as the SS 433 and the Cyg X-1 systems. Then, we concentrate on predictions of the associated emissions, focusing on detectable high-energy neutrinos and $\gamma$-rays originated from the extragalactic M33 X-7 system, which is an X-ray binary discovered in 2006, located in the neighboring galaxy Messier 33, and has not yet been modeled in detail. The particle and radiation energy distributions, produced from magnetized hadronic astrophysical jets in the context of our method, are assumed to originate from decay and scattering processes taking place among the secondary particles created when hot (relativistic) protons of the jet scatter on thermal (cold) ones (p-p interaction mechanism inside the jet). These distributions are computed by solving the system of coupled integrodifferential transport equations of multiparticle processes (reactions chain) following the inelastic proton-proton ( $p-p)$ collisions. For the detection of such high-energy neutrinos as well as multiwavelength (radio, X-ray, and gamma-ray) emissions, extremely sensitive space telescopes and other $\gamma$-ray and neutrino detection instruments are in operation or have been designed like the CTA, IceCube, ANTARES, KM3NeT, and IceCube-Gen-2.
\end{abstract}

\section{Introduction}

During the last few decades, collimated astrophysical outflows have been observed to emerge from a wide variety of galactic and extragalactic compact structures Mirabel_ Rodriguez_1999, Romero_Torres_et_al_2003, Reynoso08, Reynoso09, RomeroReview. Among such objects, the stellar scale class of microquasar (MQ) and X-ray binary systems (XRBs) possesses prominent positions [1-5]. These twobody cosmic structures consist of a collapsed stellar remnant, a stellar-mass black hole or a neutron star (compact object), and a companion (donor) main-sequence star in coupled orbit around their center of mass.
Due to the strong gravitational field pertaining around the compact object, mass from the companion star is accreted onto the equatorial region of the black hole forming an accretion disc. In the cases when the black hole is rotating rapidly and the accretion disc is geometrically rather thick and hot, ejection of two powerful oppositely directed mostly relativistic mass outflows (jets) occurs perpendicular to the accretion disk [6]. Such systems constitute excellent "laboratories" for the investigation of astrophysical outflows (jets). Currently, for the detection of multiwavelength (radio, X-ray, and gamma-ray) as well as high-energy neutrino emissions, sensitive detection instruments are in operation or have been designed like the IceCube, ANTARES, KM3NeT, CTA, and IceCube-Gen2 [7-13]. 
From a theoretical and phenomenological viewpoint, due to the presence of rather strong magnetic fields, the MQ jets are treated as magnetohydrodynamical flows emanating from the vicinity of the compact object (usually a stellar-mass black hole) [14-16], and this is also assumed to be the case in our present work. From the observed characteristics of MQs, researchers have concluded that they share a lot of similarities in their physical properties with the class of Active Galactic Nuclei (AGN), which are mostly located at the central region of galaxies. The latter, however, is enormously larger in scale compared to microquasars, and in addition, the evolution of AGNs is appreciably slower than that of MQs that makes the observation of many phenomena significantly difficult [17-22].

In this article, we focus on the magnetized astrophysical outflows (jets) that are characterized by hadronic content ( $p$, $\pi^{ \pm}$, light nuclei, etc.) in their jets. We consider them as fluid flows emanating from a central source at the jet's origin [4, $5,23]$. We, furthermore, assume that the compact object is a spinning black hole with mass up to few tens (30-50) of the Sun's mass $[1,5,24]$.

Initially, we make an effort to improve the model employed to perform numerical simulations for particle and radiation emissions from hadronic MQ jets $[25,26]$. We mention that some authors employ leptohadronic models which consider that primary electrons (and/or positrons) carry significant portion of the kinetic power inside the jets (see Section 2) [6, $24,27]$. In this paper, we adopt that the main mechanism producing the high-energy band of the electromagnetic spectrum (GeV-TeV $\gamma$-rays) and high-energy neutrinos is the protonproton ( $\mathrm{p}-\mathrm{p}$ ) collision taking place within the hadronic jets, i.e., the inelastic scattering of hot (nonthermal) protons on thermal (cold) ones [24].

The scattering, diffusion, decay, etc., of the secondary particles $\left(\pi^{ \pm}, K^{ \pm}, \mu^{ \pm}, e^{ \pm}\right.$, etc.) produced afterwards, from a mathematical modeling point of view, are governed by a system of coupled integrodifferential transport equations. One of the purposes of this work is to attempt, for a first time, to formulate in a compact way this differential system of transport equations (satisfied by the primary protons and the secondary multiparticles, multispecies) and derive advantageous algorithms [28] to perform the required simulations for persistent emissions (transient episodes or flares are ignored [6]) emanating from the hadronic component of the MQ jets. These calculations are based on the particle distributions extracted from the solutions of the time-independent (steady-state approximation) system of integrodifferential transport equations mentioned above [29-32].

Moreover, we extend the calculations of References [5, 14-16] so as to include contributions to neutrino emissivity (intensity) originating from the secondary muons $\left(\mu^{ \pm}\right)$produced from the decay of charged pions $\left(\pi^{ \pm}\right)$which in our previous works had been ignored due to the long time consuming required for such simulations. Obviously, the mathematical problem of such a study becomes also more complicated (e.g., the number of coupled equations in the abovementioned differential system increases rapidly in the case of considering neutrinos coming out of kaon $\left(K^{ \pm}\right)$decays) Lipari07.
After fixing the model parameters and testing the derived algorithms on the reproducibility of some known properties of the well-studied SS 433 galactic microquasar [14-16], we perform detailed simulations for the galactic Cyg X-1 system as well as for the extragalactic M33 X-7 MQ [33]. The latter system has not been studied up to now from a jet emission viewpoint because this is a rather recently discovered (in 2006) X-ray binary system located in the neighboring galaxy of our Milky Way Galaxy, known as Messier 33 [33].

The rest of the paper is organized as follows. In Section 2, we describe briefly the main characteristics of hadronic jets in galactic microquasars (SS433 and Cyg X-1) as well as the extragalactic M33 X-7 system. Then, in Section 3, we present the formulation of our improved method related to the differential system of transport equations (integrodifferential system of coupled equations) and derive the appropriate algorithms. In Section 4, we present and discuss our results referred to high-energy neutrino and $\gamma$-ray energy spectra emitted from the extragalactic M33 X-7 MQ. Finally, in Section 5, we summarize the main conclusions extracted from the present investigation.

\section{Brief Description of the Hadronic MQ Systems}

In this section, we summarize briefly some basic properties of the hadronic MQ systems (like those mentioned before) and the characteristics of hadronic models that describe reliably microquasar jet emissions in the high-energy band of the electromagnetic spectrum (GeV-TeV gamma-rays) as well as high-energy neutrino emission $[1,4-6]$. In the last few decades, from the investigation of a great number of MQs and X-ray emitting binary systems, stellar-mass black holes have been observed, with masses in the region of our interest determined mainly from the dynamics and structure properties of their companion stars $[17,18,20]$.

The galactic binary system SS 433, located $5.5 \mathrm{kpc}$ from the Earth in Aquila constellation, displays two mildly relativistic jets (with bulk velocity $v_{b} \approx 0.26 \mathrm{c}$ ) that are oppositely directed and precess in cones [34]. This system consists of a compact object (black hole) and an A-type companion (donor) star in coupled orbit with a period $P \sim 13.1$ days [21]. For the masses of the component stars of this system, in this work, we adopted the observations of the INTEGRAL [22], i.e., $M_{\mathrm{BH}}=9 M_{\odot}$ and $M_{\text {don }}=30 M_{\odot}$ for the black hole and the companion star, respectively. According to recent estimations for SS433, it suggests a low limit of the compact object of $5-9 M_{\odot}$ placing it as a stellar-mass black hole [19].

The second galactic system addressed in this work, Cygnus $\mathrm{X}-1$, is an $\mathrm{X}$-ray source in the constellation Cygnus which is located $1.86 \mathrm{kpc}$ from the Earth [3]. The $19.2 M_{\odot}$ O-type companion star [35] is in coupled orbit with the $14.8 M_{\odot}$ compact object (stellar-mass black hole) [35], with orbital period $P \sim 5.6$ days [36].

As mentioned before, one of our main goals in this work is to calculate high-energy $\gamma$-ray and neutrino emissions from the binary system M33 X-7 [37, 38] which is located 
in the nearby galaxy Messier 33, the only known up to now black hole that is in an eclipsing binary system, with orbital period $P \sim 3.45$ days [33]. The distance from Earth of this system is between $\sim 840 \mathrm{kpc}$ [39] and $\sim 960 \mathrm{kpc}$ [40]. In addition, the black hole mass is $M_{\mathrm{BH}}=(15.65 \pm 1.45) M_{\odot}$ which orbits its $M_{\text {don }}=(70.0 \pm 6.9) M_{\odot}$ O-type companion star.

In Table 1, we tabulate in addition some other important parameters employed in this work for the systems under investigation, SS 433, Cygnus X-1, and M33 X-7.

2.1. Dynamics and Energetic Evolution of Hadronic MQ Jets. In the model considered in this work for the description of MQ jets, an accretion disk in the equatorial region of the compact object is present, and a fraction of the accreted material is expelled in two oppositely directed jets [4, 5, 41]. We assume an approximately conical mass outflow (jet) with a half-opening angle $\xi$ (for the M33 X-7 MQ, we adopt the value $\xi=7^{\circ}$ ) and a radius given by $r(z)=z \tan \xi$ (the coordinate $z$-axis coincides with the cone axis, jet's direction). The injection point (plane) of the jet is at a distance $z_{0}$ from the compact object. When $z=z_{0}$, the radius of the jet is given by $r_{0}=z_{0} \tan \xi$ (see Table 1 ).

The initial jet radius is $r_{0}=r\left(z_{0}\right) \approx 5 R_{\text {sch }}$, where $R_{\text {sch }}=2$ $G M_{\mathrm{BH}} / c^{2}\left(R_{\mathrm{sch}}\right.$ is the known Schwarzschild radius, the critical black hole radius for which the escape speed of particles becomes equal to the speed of light $c$ ), with $G$ denoting Newton's gravitational constant. Then, we find, for example, that the injection point is at $z_{0}=r_{0} / \tan \xi \simeq 1.9 \times 10^{8} \mathrm{~cm}$, for the M33 X-7. For the sake of comparison, we mention that for Cygnus $\mathrm{X}-1$, the injection point is at $z_{0} \simeq 10^{8} \mathrm{~cm}$, while for SS 433 , it is $z_{0} \simeq 1.3 \times 10^{9} \mathrm{~cm}$.

It should be also noted that, for all systems studied in this work, the extension of the jet is determined through a maximum value of $z$, denoted by $z_{\max }$, which is assumed to be equal to $z_{\max }=5 z_{0}$ (this point may be assumed to be at the boundaries of the jet with the ambient region).

In discussing the energetic evolution and dynamics of MQ jets, the kinetic energy density of the jet, $\rho_{k}(z)$, is related to its kinetic luminosity, $L_{k}$, through the expression [1]

$$
\rho_{k}(z)=\frac{L_{k}}{\pi v_{b}[r(z)]^{2}},
$$

where $v_{b}$ is the bulk velocity of the jet particles (mostly protons). Within the context of the jet-accretion coupling hypothesis, only around $10 \%$ of the Eddington luminosity goes into the jet [42]. Here, we adopt $L_{k}=10^{38} \mathrm{erg} \mathrm{s}^{-1}$, for a black hole of mass $\sim 15.7 M_{\odot}$, i.e., for the M33 X-7 system.

Furthermore, assuming equipartition between the magnetic energy and the kinetic energy in the jet, it implies that $\rho_{\text {mag }}=\rho_{k}[43,44]$, and hence, the magnetic field that collimates the astrophysical jet plasma is given by [27]

$$
B(z) \equiv \sqrt{8 \pi \rho_{\mathrm{mag}}(z)}=\sqrt{8 \pi \rho_{k}(z)}
$$

In general, for the kinetic power in the jet, many authors consider that a fraction is carried by primary pro- tons and the rest is carried by electrons, i.e., $L=L_{p}+L_{e}$. Then, the relation between the proton and electron power is determined through a parameter $\alpha$ in such a way that $L_{p}=\alpha L_{e}[6,24,27]$.

In this article, however, we adopt the case of $\alpha=100$ which means that we consider a proton-dominated jet. Other quantities needed for the purposes of this paper are listed in Table 1.

2.2. $p$ - $p$ Collision Mechanism inside MQ Jets. The collision of relativistic protons with the cold ones inside the jet ( $\mathrm{p}-\mathrm{p}$ collision mechanism) produces high-energy charged particles (pions $\pi^{ \pm}$, kaons $K^{ \pm}$, muons $\mu^{ \pm}$, etc.) and neutral particles $\left(\pi^{0}, K^{0}, \tilde{K}^{0}, \eta\right.$ particles, etc.). Important primary reactions of this type are

$$
\begin{aligned}
& p+p \longrightarrow p+p+a \pi^{0}+b\left(\pi^{+}+\pi^{-}\right) \\
& p+p \longrightarrow p+n+\pi^{+}+a \pi^{0}+b\left(\pi^{+}+\pi^{-}\right), \\
& p+p \longrightarrow n+n+2 \pi^{+}+a \pi^{0}+b\left(\pi^{+}+\pi^{-}\right)
\end{aligned}
$$

where $a$ and $b$ denote the pion multiplicities [1], and similarly for kaon production [45].

Charged pions $\pi^{ \pm}$(and kaons $K^{ \pm}$), afterwards, decay to charged leptons (muons $\mu^{ \pm}$, and electrons or positrons, $e^{ \pm}$) as well as neutrinos and antineutrinos as

$$
\begin{aligned}
& \pi^{+} \longrightarrow \mu^{+}+v_{\mu}, \quad \pi^{+} \longrightarrow e^{+}+v_{e} \\
& \pi^{-} \longrightarrow \mu^{-}+\bar{v}_{\mu}, \quad \pi^{-} \longrightarrow e^{-}+\bar{v}_{e} .
\end{aligned}
$$

Furthermore, muons also decay giving neutrinos and electrons (or positrons) as

$$
\mu^{+} \longrightarrow e^{+}+v_{e}+\bar{v}_{\mu}, \quad \mu^{-} \longrightarrow e^{-}+\bar{v}_{e}+v_{\mu} .
$$

We mention that neutral pions $\left(\pi^{0}\right), \eta$-particles, etc., decay producing $\gamma$-rays according to the reactions

$$
\begin{gathered}
\pi^{0} \longrightarrow \gamma+\gamma, \quad \pi^{0} \longrightarrow \gamma+e^{-}+e^{+}, \\
\eta \longrightarrow \gamma+\gamma, \quad \eta \longrightarrow \pi^{0}+e^{-}+e^{+} .
\end{gathered}
$$

In our present study, we consider neutrinos generated from both types of charged-pion decays (for neutrinos coming from kaon decays, the reader is referred, e.g., to Reference [45] and also from both types of charged muon decays $[25,26]$. Moreover, we consider $\gamma$-rays produced from the $\pi^{0}$ decays of Equation (6). Thus, high-energy neutrinos are inevitably accompanied by pionic gamma-rays, a phenomenon well known as multimessenger emission from microquasar jets.

2.3. Accelerating and Cooling Rates of the Jet Processes. In general, the primary charged particles ( $p$ and $e^{-}$) gain energy during moving within the magnetic field $B$ (Fermi acceleration). The acceleration rate of the (initially cold) protons to 
Table 1: Parameters of the model for M33 X-7, Cygnus X-1, and SS 433.

\begin{tabular}{|c|c|c|c|c|}
\hline Parameter & Symbol & SS 433 & Cygnus X-1 & M33 X-7 \\
\hline Black hole mass & $M_{\mathrm{BH}}$ & $9.0 M_{\odot}$ & $14.8 M_{\odot}$ & $15.65 M_{\odot}$ \\
\hline Distance from Earth & $d$ & $5.5 \mathrm{kpc}$ & $1.86 \mathrm{kpc}$ & $840-960 \mathrm{kpc}$ \\
\hline Donor star mass & $M_{\text {don }}$ & $30 M_{\odot}$ & $19.2 M_{\odot}$ & $70 M_{\odot}$ \\
\hline Donor star type & - & A-type & O-type & O-type \\
\hline Orbital period & $P$ & 13.1 days & 5.6 days & 3.45 days \\
\hline Jet's kinetic power & $L_{k}$ & $10^{39} \mathrm{ergs}^{-1}$ & $10^{38} \mathrm{ergs}^{-1}$ & $10^{38} \mathrm{ergs}^{-1}$ \\
\hline Jet's launching point & $z_{0}$ & $1.3 \times 10^{9} \mathrm{~cm}$ & $10^{8} \mathrm{~cm}$ & $1.9 \times 10^{8} \mathrm{~cm}$ \\
\hline Bulk velocity of jet particles & $v_{b}$ & $0.26 \mathrm{c}$ & $0.6 \mathrm{c}$ & $0.8 \mathrm{c}$ \\
\hline Jet's bulk Lorentz factor & $\Gamma_{b}$ & 1.04 & 1.25 & 1.66 \\
\hline Jet's half-opening angle & $\xi$ & $0.6^{\circ}$ & $1.5^{\circ}$ & $7^{\circ}$ \\
\hline Jet's viewing angle & $\theta$ & $78.05^{\circ}$ & $27.1^{\circ}$ & $74.6^{\circ}$ \\
\hline
\end{tabular}

an energy $E=E_{p}$, known as shock acceleration, is defined as $t_{\mathrm{acc}}^{-1}=E^{-1} d E / d t$ and is given by the relation [1]

$$
t_{\mathrm{acc}}^{-1} \equiv \frac{1}{E} \frac{d E}{d t} \approx \eta \frac{c e B}{E_{p}}
$$

$\eta=0.1$ is the acceleration efficiency which means that only $10 \%$ of the cold/thermal protons may acquire relativistic energies. This is equivalent to existence of an efficient accelerator at the base of the jets where shocks are rather relativistic [46].

In the $\mathrm{p}-\mathrm{p}$ collision mechanism, the cross section and the rate of inelastic p-p scattering (relativistic protons scatter on cold ones), $\sigma_{p p}^{\text {inel }}$ and $t_{p p}^{-1}$, respectively, play a crucial role (the inelasticity coefficient is $K_{p p} \approx 1 / 2$ ). The corresponding cross section, $\sigma_{p p}^{\text {inel }}$, as a function of the fast proton energy $E_{p}$, reads $[47,48]$

$$
\sigma_{p p}^{\text {inel }}\left(E_{p}\right)=\left(0.25 L^{2}+1.88 L+34.3\right)\left[1-\left(\frac{E_{\mathrm{th}}}{E_{p}}\right)^{4}\right]^{2} \times 10^{-27} \mathrm{~cm}^{2} \text {, }
$$

where $L=\ln \left(E_{p} / 1000 \mathrm{GeV}\right)$ and $E_{\text {th }}$ denotes the minimum energy of (thermal) protons, $E_{p}^{\mathrm{min}}$, which is equal to $E_{p}^{\min } \equiv E_{\mathrm{th}}=1.22 \mathrm{GeV}$, see e.g., Appendix of Reference [5].

The corresponding rate, $t_{p p}^{-1}$, of the inelastic $\mathrm{p}$-p scattering is given in Table 2 in terms of the cross section $\sigma_{p p}^{\text {inel }}$ and the number density of cold particles (jet protons), $n_{p}(z)$, at a distance $z$ from the black hole which may be written as [1]

$$
n_{p}(z)=\frac{\left(1-q_{\mathrm{rel}}\right)}{\Gamma_{b} m_{p} c^{2}} \rho_{k}(z)
$$

$q_{\text {rel }}=0.1$ denotes the portion of the relativistic protons.

In Table 2 , in addition to the $\mathrm{p}$ - $\mathrm{p}$ collision rate and accelerating rates of protons, we tabulate the most significant cooling rates (synchrotron, adiabatic, etc.) for protons, pions, and muons as well.

In more detail, the well-known synchrotron radiation is emitted from charged particles of energy $E=\gamma m c^{2}$, with $\gamma$ being the particle's Lorentz factor and $m$ its mass, moving inside the magnetized plasma. Thus, the rate of the synchrotron radiation, $t_{\text {syn }}^{-1}$, is very important in MQ systems with strong magnetic fields (see Table 2).

Furthermore, due to the adiabatic expansion of the jet plasma, all comoving particles inside it lose energy with a rate $t_{\mathrm{ad}}^{-1}[44]$. This rate, known as the adiabatic cooling rate, depends on the particle velocities $v_{b} \tan \xi$.

In addition, $t_{\pi p}^{-1}$ gives the rate of pion-proton $(\pi-p)$ inelastic scattering inside the jet. The corresponding $\sigma_{\pi p}^{\text {inel }}$ cross section is related to the inelastic p-p scattering cross section with the relationship $\sigma_{\pi p}(E)=(2 / 3) \sigma_{p p}^{\text {inel }}(E)$, where the factor $2 / 3$ comes out of the fact that protons are made of three valence quarks, while the pions of only two quarks [49].

It is worth mentioning that some processes taking place inside the jet lead to particles' knock out, either by escaping from the jet (and/or from the energy range of interest) or by their decay processes [50]. Thus, the corresponding rates are (i) the escaping rate, $t_{\mathrm{esc}}^{-1}$, given by

$$
t_{\mathrm{esc}}^{-1} \approx \frac{c}{z_{\max }-z}
$$

$z_{\max }-z_{0}$ represents the length of the acceleration zone, and (ii) the decay rate of the particle in question, $t_{\mathrm{dec}}^{-1}$, which is known from direct measurements of particle's life time.

The rates of the knock out (or catastrophic) processes inside the jet for pions, $t_{\pi}^{-1}$, and muons, $t_{\mu}^{-1}$, include contributions from the decay process and escaping process as

$$
\begin{gathered}
t_{\pi}^{-1}(E, z)=t_{\mathrm{esc}, \pi}^{-1}(z)+t_{\mathrm{dec}, \pi}^{-1}(E), \\
t_{\mu}^{-1}(E, z)=t_{\mathrm{esc}, \mu}^{-1}(z)+t_{\mathrm{dec}, \mu}^{-1}(E),
\end{gathered}
$$


TABLE 2: Accelerating, cooling, and decay rates for particles moving inside hadronic MQ jets.

\begin{tabular}{lcc}
\hline Rate parameter & $\begin{array}{c}\text { Rate } \\
\text { symbol }\end{array}$ & Basic rate definition \\
\hline Proton accelerating rates & $t_{\mathrm{acc}}^{-1}$ & $\eta\left(c e B / E_{p}\right)$ \\
$\begin{array}{l}\text { Proton-proton collision } \\
\text { rate }\end{array}$ & $t_{p p}^{-1}$ & $n_{p}(z) \sigma_{p p}^{\mathrm{inel}}\left(E_{p}\right) K_{p p}$ \\
$\begin{array}{l}\text { Synchrotron radiation } \\
\text { rate }\end{array}$ & $t_{\mathrm{syn}}^{-1}$ & $4 / 3\left(m_{e} / m\right)^{3}\left(\gamma \sigma_{T} B^{2} / m_{e} c 8 \pi\right)$ \\
Adiabatic expansion rate & $t_{a d}^{-1}$ & $2 / 3\left(v_{b} / z\right)$ \\
Pion-proton collision & $t_{\pi p}^{-1}$ & $0.5 c n_{p}(z) \sigma_{\pi p}^{\text {inel }}\left(E_{p}\right)$ \\
$\begin{array}{l}\text { Proton knock out } \\
\text { (escape) rate }\end{array}$ & $t_{p}^{-1}=t_{\mathrm{esc}, p}^{-1}$ & $t_{\mathrm{esc}, p}^{-1}=c /\left(z_{\mathrm{max}}-z\right)$ \\
Pion knock out rate & $t_{\pi}^{-1}$ & $t_{\mathrm{esc}, \pi}^{-1}+t_{\mathrm{dec}, \pi}^{-1}$ \\
Muon knock out rate & $t_{\mu}^{-1}$ & $t_{\mathrm{esc}, \mu}^{-1}+t_{\mathrm{dec}, \mu}^{-1}$ \\
\hline
\end{tabular}

where $t_{\mathrm{dec}, \pi}^{-1}=\left[\left(2.6 \times 10^{-8}\right) \gamma_{\pi}\right]^{-1} s^{-1}$, for pions, and $t_{\mathrm{dec}, \mu}^{-1}=$ $\left[\left(2.2 \times 10^{-6}\right) \gamma_{\mu}\right]^{-1} s^{-1}$, for muons. We note that Equation (12) holds also for protons due to the fact that $t_{\mathrm{dec}, p}^{-1}=0$ (or equivalently the proton life time is infinite), so as $t_{p}^{-1}(E, z)=t_{\text {esc }, p}^{-1}(z)$, see Table 2 .

The rates discussed above enter the set of basic coupled transport equations (system of kinematic equations) that describe the particle distributions as we will discuss in Section 3.

\subsubsection{Energy Variation of the Various Rates of Jet Particles.} In Figures 1-3, the variation of the aforementioned main rates, versus the particle energy $E$, for protons, pions, and muons, respectively, and for the SS 433 and M33 X-7 MQ systems, is illustrated. In other words, in these figures, the region of dominance of the accelerating, cooling, etc., rates throughout the energy range of interest, $E_{\text {th }} \equiv E^{\min } \leq E \leq$ $E^{\max }$, is demonstrated.

More specifically, Figure 1 indicates the cooling rates at the base of the jet for protons, Figure 2 shows those for pions and Figure 3 shows the corresponding ones for muons, in the extragalactic binary system M33 X-7 (top of these figures). For comparison, the corresponding results for the galactic binary system SS 433 (bottom of these figures) are also shown.

The plots in Figure 2 for pions, show, respectively, the variation versus the energy $E$ of the rate for synchrotron emission (solid lines), for the pion-proton inelastic collision (dashed lines), for the adiabatic cooling (dotted lines), and for the decay of pions (dot-dashed lines). They refer to the M33 X-7 (top) and the SS 433 (bottom) microquasar systems. Also, Figure 3 illustrates the cooling rates of muons for the synchrotron emission (solid lines), for the adiabatic expansion (dotted lines), and for the decay of muons (dot-dashed lines). They are similar to those for pions (Figure 2) except for the corresponding muon-proton and muon-pion scattering channels which are ignored in this work.
From Figures 2 and 3, it becomes obvious that the particle synchrotron losses dominate the high-energy region. In the case of protons (Figure 1), however, due to the large proton mass, synchrotron losses are not dominant up to very high energies $\left(\sim 10^{7} \mathrm{GeV}\right)$ for the MQ systems studied. On the other hand, for pions and muons, the decay losses dominate for lower energies, due to their short life time (see Table 2).

The main difference between these two MQ systems (SS 433 and M33 X-7) concerns the synchrotron cooling rates. In general, according to Equations (1) and (2), for systems with wide half-opening angle (e.g., M33 X-7, with $\xi=7^{\circ}$ ), the magnetic energy density is lower than that of systems with small $\xi$ (e.g., the SS 433 with $\xi=0.6^{\circ}$ ). Hence, the magnetic field for M33 X-7 is lower which becomes obvious by comparing Equation (2) for the two systems. The latter conclusion justifies the lower synchrotron loss rate for the M33 $\mathrm{X}-7$ system.

\section{Formulation of the Key-Role Transport Equations inside Jet Plasmas}

In this section, we make a first step towards improving the mathematical formulation describing the creation, scattering, decay, diffusion, and emission of particles (including neutrinos) and electromagnetic radiation in astrophysical outflows (jets). The basic key-role tool of this formalism is the general transport equation which satisfies each of the particles considered [6].

We should note that such a formalism may be reliably applicable for multispecies (multi-particle) emissions from MQs and X-ray binaries, as well as from central regions of galaxies like the AGN systems, in which the compact object is a massive or supermassive black hole, see, e.g., $[1,50]$. The geometry of the latter sources is assumed to be rather spherical, while in the first class of systems that are studied in this work, the sources are assumed conical and the particles (neutrinos) or radiation are emitted to the forward or backward direction.

From a mathematical point of view, the transport equation which satisfies any kind of particles moving into the (dark) jets of MQs is an integrodifferential equation, as is explained the following.

3.1. The Transport Equation for Particles Moving inside MQ Jets. The general transport equation describes the concentration (distribution) of particle of $j$-kind, $N_{j}(E, r, t)$, where $j=p, e^{ \pm}, \pi^{ \pm}, \mu^{ \pm}$, etc., as a function of the time $t$, the particle's energy $E$, and the position $r$ inside the (conical) jet. In essence, this is a phenomenological macroscopic equation of particle (or radiation) transport describing astrophysical outflows, and it is written as

$$
\begin{aligned}
& \frac{\partial N_{j}}{\partial t}-\nabla \cdot\left(D_{j} \nabla N_{j}\right)+\frac{\partial\left(b_{j} N_{j}\right)}{\partial E}-\frac{1}{2} \frac{\partial^{2}\left(d_{j} N_{j}\right)}{\partial E^{2}}=Q_{j}(E, \mathbf{r}, t) \\
& -p_{j} N_{j}+\sum_{k} \int P_{j}^{k}\left(E^{\prime}, E\right) N_{j}(E, \mathbf{r}, t) d E, \quad j=p, \pi^{ \pm}, \mu^{ \pm} \cdots,
\end{aligned}
$$




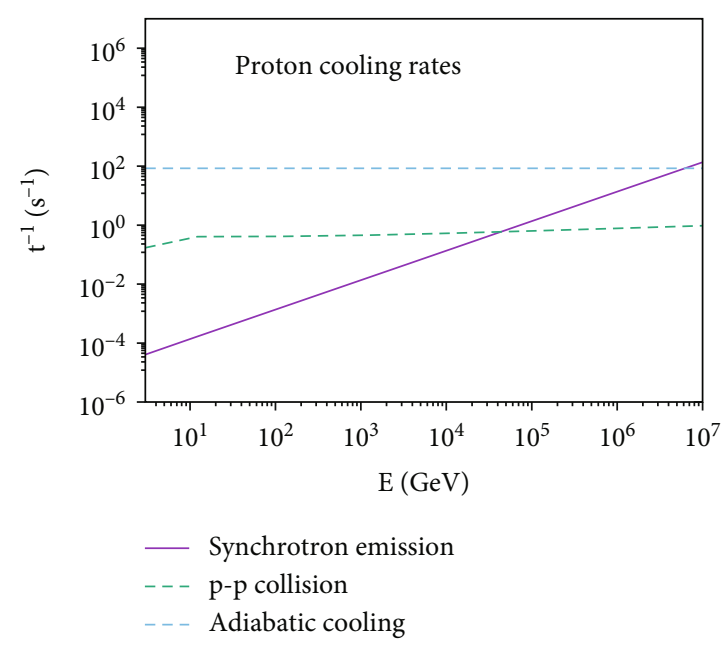

(a)

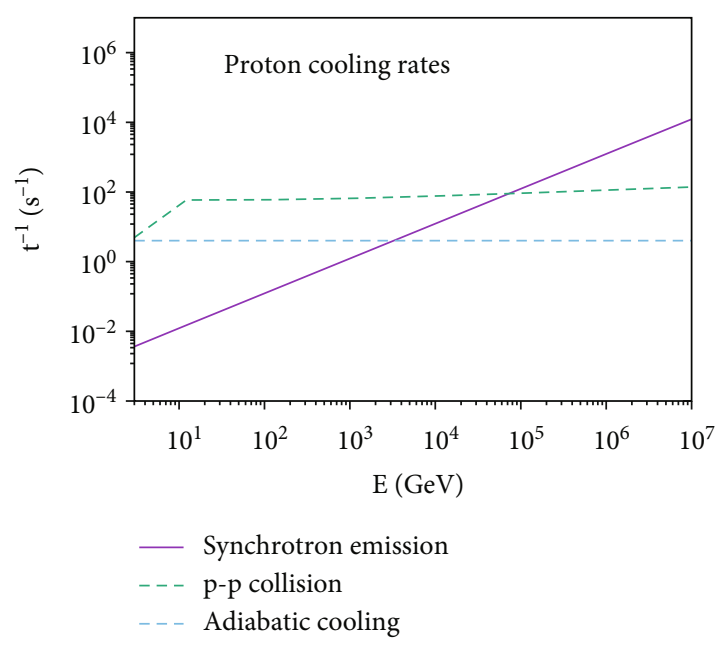

(b)

FIGURE 1: Cooling rates for protons in the jets of M33 X-7 (a) and SS 433 (b) at the base of the jets $z_{0}$. The plots of cooling rates show the synchrotron emission (solid lines), the adiabatic cooling (dotted lines), and the inelastic p-p collision rate (dashed lines).

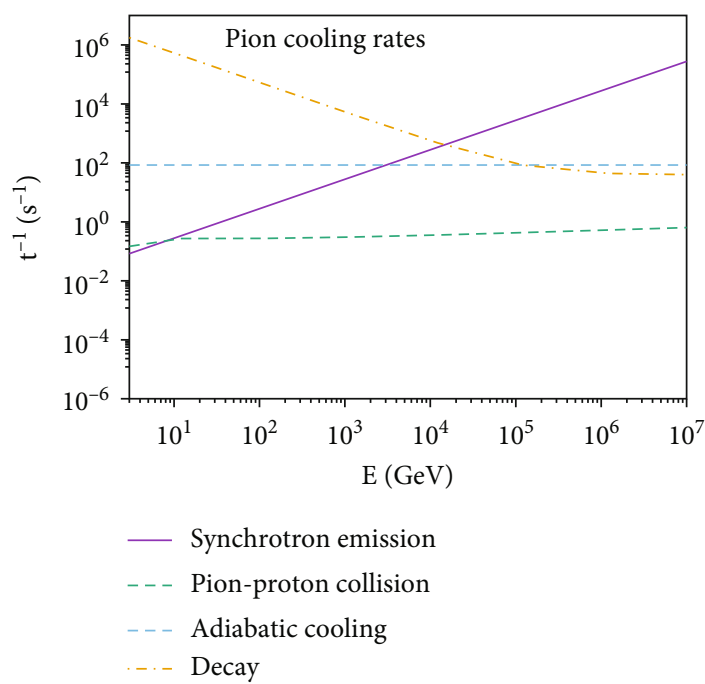

(a)

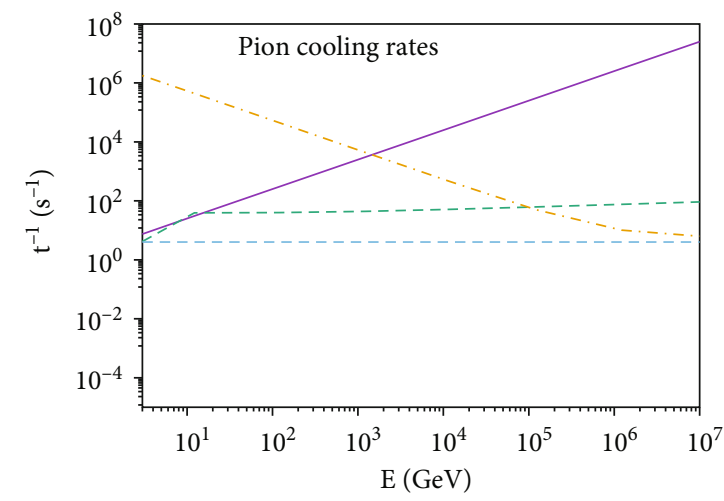

_ Synchrotron emission
$\ldots$ - Pion-proton collision
$\ldots$ _- Adiabatic cooling
$\ldots$-.- Decay

(b)

FIGURE 2: Cooling rates for pions in the jets of M33 X-7 (a) and SS 433 (b) at the base of the jets.

where the parameters $D_{j}, b_{j}, d_{j}, p_{j}$, and $P_{j}^{k}$ may depend upon the space and time coordinates and also on the energy $E$. The latter equation coincides with the continuity equation for particles of type- $j$, with $j=1,2,3$ and $(1,2,3) \equiv(p, \pi, \mu)$.

The term $Q_{j}(E, \mathbf{r}, t)$ in the r.h.s. of Equation (14) is equal to the intensity of the source producing the particles- $j$, which is also known as the injection function of particles $-j$. This means that $Q_{j}(E, \mathbf{r}, t) d E d^{3} \mathbf{r} d t$ represents the number of particles kind- $j$ provided by the sources in a volume element $d^{3} \mathbf{r}$, in the energy range between $E$ and $E+d E$ during the time $d t$. In the case when the $j$-type particles are products of a chain reaction (as it holds in our present work assuming the p-p reaction chain described in Section 2.2), the function $Q_{j}(E, \mathbf{r}, t)$ couples the $j$-reaction with its parent reaction, i.e., the equation of particles kind- $(j-1)$.

Further, the term proportional to $p_{j}$ exists in cases when catastrophic (or knock out) processes take place that cause catastrophic energy losses. Then, this gives the probability per unit time for the losses in question to occur. Thus, the coefficient $p_{j}$ is written as

$$
p_{j}=\frac{1}{T_{j}} \equiv t_{j}^{-1},
$$

with $T_{j}$ is the mean life time of the particles of kind- $j$. 


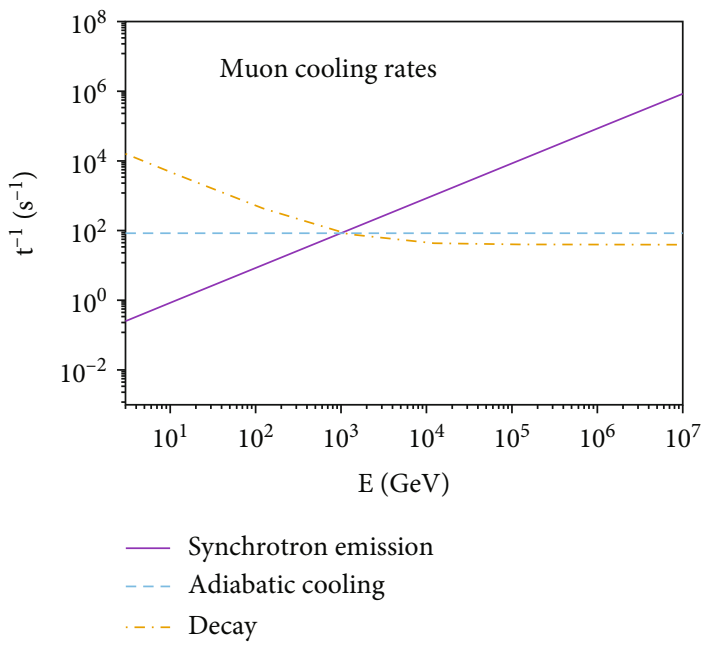

(a)

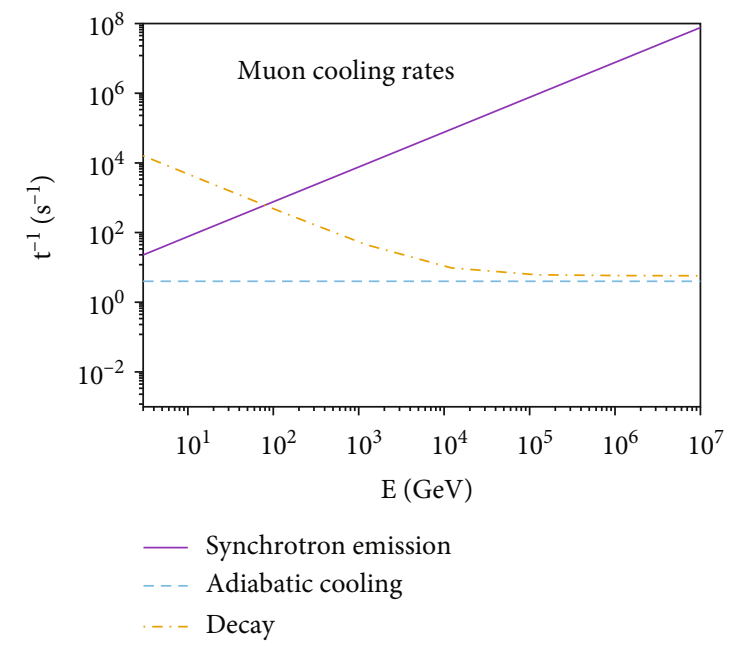

(b)

FIgURE 3: Cooling rates for muons in the jets of M33 X-7 (a) and SS 433 (b) at the base of the jets.

Also, $p_{j} N_{j}$ denotes the number of particles "knocked out" per unit time.

The coefficients $b_{j}$ in the third term (r.h.s.) are equal to the mean energy increment of the particle- $j$ per unit time, i.e.,

$$
b_{j}=\frac{d E}{d t} .
$$

For the special case when $b_{j}=b_{j}(E)$, this coefficient is related to energy losses of various cooling processes. Also, the coefficients $P_{j}^{k}(E)$, with $k>j$, in the latter summation of the transport equation, are related to the existence of fragmentation of the primary (or secondary) particles which in this work are assumed as nonexisting.

The parameter $D_{j}$ is known as the diffusion coefficient which, in general, is a function of the coordinates $\mathbf{r}$ and time $t$ if the concentration of the jet plasma and its macroscopic motion are inhomogeneous in the volume of the jet. By assuming that $D_{j}=0$, we may reliably describe the regular motion of particles $j$-kind along the lines of force of the magnetic field $B$.

For simplicity, in this work, we make the realistic assumptions that the above coefficients depend only on the particle's energy $E$, i.e., we assume that $D_{j}(E), b_{j}(E)$, and $p_{j}(E)$. Then, the system of general transport equations (14) reads

$$
\begin{gathered}
\frac{\partial}{\partial t}\left(\begin{array}{c}
N_{p}(E, z, t) \\
N_{\pi}(E, z, t) \\
N_{\mu}(E, z, t)
\end{array}\right)-\nabla^{2}\left(\begin{array}{c}
D_{p} N_{p}(E, z, t) \\
D_{\pi} N_{\pi}(E, z, t) \\
D_{\mu} N_{\mu}(E, z, t)
\end{array}\right)+\frac{\partial}{\partial E}\left(\begin{array}{c}
b_{p} N_{p}(E, z, t) \\
b_{\pi} N_{\pi}(E, z, t) \\
b_{\mu} N_{\mu}(E, z, t)
\end{array}\right) \\
\quad-\frac{1}{2} \frac{\partial^{2}}{\partial E^{2}}\left(\begin{array}{c}
d_{p} N_{p}(E, z, t) \\
d_{\pi} N_{\pi}(E, z, t) \\
d_{\mu} N_{\mu}(E, z, t)
\end{array}\right)+\left(\begin{array}{c}
t_{e s c}^{-1} N_{p}(E, z, t) \\
t_{\pi}^{-1} N_{\pi}(E, z, t) \\
t_{\mu}^{-1} N_{\mu}(E, z, t)
\end{array}\right)=\left(\begin{array}{c}
Q_{p}(E, z, t) \\
Q_{\pi}(E, z, t) \\
Q_{\mu}(E, z, t)
\end{array}\right) .
\end{gathered}
$$

Note that, in the latter system of coupled differential equations, we write down only three particles (protons, pions, and muons), without considering the complete reaction family tree, since we have not distinguished particles with different charge as $\pi^{ \pm}$and $\mu^{ \pm}$, and we have not considered the possibility of left-right symmetry, $\mu_{L, R}$, of muons as we have done in Section 4 . This means that, by considering all these particles, the system of Equation (17) will have seven lines.

Usually, we ignore the term involving second derivative with respect to $E$ means that the acceleration term is negligible, an approximation adopted even by recent leptohadronic steady-state models [31].

The solution of the general transport equation for particles of one kind (when the last term of this equation can be omitted) is described in detail in Reference [16]. In the latter work, however, neutrinos produced only from $\pi^{ \pm}$have been considered. In the present paper, we proceed further and include neutrino emissions also from the muon decays, so we need to solve the system of Equation (17) by including the third line too.

The complete form of the system of Equation (17) is treated with the method of Reference [28] in order to find the exact solutions. In Reference [26], Equation (17) is treated semianalytically. In the present work, we restrict ourselves to simplified forms resulting by neglecting various phenomena (processes) taking place inside the astrophysical outflows (jet plasma), even though some of the assumed omissions may be considered as rather crud approximations.

In the following, we discuss the cases of Equation (17) satisfying the conditions of the one-zone approximation $[16,51]$, i.e., the cases when the particle distributions are independent of time (steady-state approximation).

3.1.1. Transport Equation Assuming Absence of Energy Losses. At first, in the calculations of our present work, we start by writing the simplest solutions of the system of transport equations obtained by assuming that all energy losses are absent, i.e., $b_{j}=0$. Then, by denoting $N_{p, 0}, N_{\pi, 0}$, and 
$N_{\mu, 0}$ the corresponding energy distributions for protons, pions, and muons, respectively, the system of transport equations takes the trivial form

$$
\left(\begin{array}{c}
t_{p}^{-1}(E) N_{p, 0}(E, z) \\
t_{\pi}^{-1}(E) N_{\pi, 0}(E, z) \\
t_{\mu}^{-1}(E) N_{\mu, 0}(E, z)
\end{array}\right)=\left(\begin{array}{c}
Q_{p}(E, z) \\
Q_{\pi}(E, z) \\
Q_{\mu}(E, z)
\end{array}\right)
$$

The calculated distributions $N_{j, 0}$, with $j=p, \pi^{ \pm}$, and $\mu^{ \pm}$ of the latter equations are discussed in the next section.

3.1.2. Steady-State Transport Equations with Particle Losses when Moving inside the Jet Plasma. Under the conditions of the steady-state approximation, and assuming that the various energy losses are absent, the system of transport equations (Equation (17)) takes the form

$$
\frac{\partial}{\partial E}\left(\begin{array}{c}
b_{p} N_{p}(E, z) \\
b_{\pi} N_{\pi}(E, z) \\
b_{\mu} N_{\mu}(E, z)
\end{array}\right)+\left(\begin{array}{c}
t_{e s c}^{-1} N_{p}(E, z) \\
t_{\pi}^{-1} N_{\pi}(E, z) \\
t_{\mu}^{-1} N_{\mu}(E, z)
\end{array}\right)=\left(\begin{array}{c}
Q_{p}(E, z) \\
Q_{\pi}(E, z) \\
Q_{\mu}(E, z)
\end{array}\right) .
$$

In order to calculate the time-independent neutrino and gamma-ray emissivities, we need, first, to calculate the distributions of protons, pions, and muons, $N_{p}, N_{\pi}$, and $N_{\mu}$, respectively, from Equation (19). For these computations, we used a code written in the $\mathrm{C}$ programming language, mainly following the assumptions of References [6, 24, 27].

We note that, in the 1.h.s. of Equation (19), the rates of the knock out processes inside the jet, $t_{j}^{-1}$, for the particles $j=p, \pi, \mu$, are provided by Equation (12) and the values given below that equation. Moreover, the coefficients $b_{j}$ entering Equation (19) are obtained from expressions of the form $b_{j}(E)=-E t_{\text {loss }, j}^{-1}(E)$ by inserting the corresponding energy loss rates $t_{\text {loss }, j}^{-1}$ for each particle as

$$
\begin{aligned}
& b_{p}(E)=-E t_{\text {loss }, p}^{-1}(E)=-E\left(t_{\mathrm{syn}}^{-1}+t_{\mathrm{ad}}^{-1}+t_{p p}^{-1}\right), \\
& b_{\pi}(E)=-E t_{\mathrm{loss}, \pi}^{-1}(E)=-E\left(t_{\mathrm{syn}}^{-1}+t_{\mathrm{ad}}^{-1}+t_{\pi p}^{-1}\right), \\
& b_{\mu}(E)=-E t_{\mathrm{loss}, \mu}^{-1}(E)=-E\left(t_{\mathrm{syn}}^{-1}+t_{\mathrm{ad}}^{-1}\right) .
\end{aligned}
$$

It is worth noting that, in the latter equations, some additional energy loss terms may appear in cases when the rates of some other processes, ignored in our present work (like, e.g., the inverse Compton scattering and the pionmuon scattering), may be considered important for the description of neutrino and $\gamma$-ray emissions from other cosmic structures (AGNs, etc.).

3.2. Brief Description of the Model Assumptions. Before embarking to concrete calculations, we find it helpful for the reader to briefly summarize the simplifications made and the main approximations assumed in our method. At first, in purely hadronic models, only the protons from the primary charged particles $\left(\mathrm{p}, e^{+}, e^{-}\right)$inside the jet are considered to contribute significantly in the emission of highenergy $\gamma$-rays and neutrinos, see References $[4,5]$ and discussion after Equation (2).

As mentioned in Introduction, in the present calculations, we consider that the $(\mathrm{GeV}-\mathrm{TeV}$ energy range of our interest) neutrinos and $\gamma$-rays are due to the $\mathrm{p}$ - $\mathrm{p}$ collision mechanism taking place inside the microquasar jets. The maximum nonthermal (primary) proton energy employed in our numerical integrations in the following (Section 3) is taken equal to $E_{p}^{\max } \approx 10^{6}-10^{7} \mathrm{GeV}$. We should stress that the $\mathrm{p}-\mathrm{p}$ mechanism is assumed to dominate over others like, e.g., scattering of jet protons with the protons of the donorstar wind or the proton-photon scattering inside the jet (case of $\gamma$-ray production) [1].

We, furthermore, note that in this work, we ignore neutrinos coming from the decay of the secondary kaons $\left(K^{ \pm}\right)$ and $\gamma$-rays produced from $\eta$ particles, neutral kaons, etc. As discussed in Section 2.2, the contributions originated from charged pion decays $\left(\pi^{ \pm}\right)$and the subsequent charged muon decays $\left(\mu^{ \pm}\right)$, for neutrino production, and those coming from neutral pion decays $\left(\pi^{0}\right)$, for $\gamma$-ray production, are assumed to be the main chain-reaction channels.

\section{Injection Functions and Energy Distributions of Particles inside the Jet}

In this section, the source functions $Q_{j}(E, \mathbf{r}, t)$ entering the r.h.s of the system of coupled integrodifferential equations of transport type are discussed. Towards this aim, initially in the system of Equation (17), we insert phenomenological expressions obtained from Reference [45]. A mathematical semianalytic way of solving this system is proposed in Reference [52]. Also, following Reference [28], a method is derived for the numerical solution of the differential system of transport equations.

\subsection{Proton Injection Function and Energy Distribution}

4.1.1. Relativistic Proton Injection Function. In the assumed $\mathrm{p}-\mathrm{p}$ mechanism, the density of fast (relativistic) protons injected is more important, while the corresponding density of slow (thermal) protons is dynamically significant.

A usual injection function for the relativistic protons, coming out of the acceleration mechanism, has been taken to be a power-law with exponent equal to two, i.e., a function of the energy of the form [53]

$$
Q_{p}(E, z)=Q_{0}\left(\frac{z_{0}}{z}\right)^{3} \frac{1}{E^{2}}
$$

where $Q_{0}$ is a normalization constant obtained by specifying the power in the relativistic protons [24], see the appendix. The above form is valid for the jet's frame of reference (the corresponding expression in the observer's system is shown in the appendix).

It should be noted that Equation (21) enters (through integration) the determination of the injection functions of 
the secondary particles $\left(\pi^{ \pm}, \mu^{ \pm}\right.$, see the following) and this justifies the term systems of coupled integrodifferential equations used in Section 3. The latter injection functions are also dependent on the rates and cross sections of the reactions that preceded in the chain processes following the inelastic p-p collision.

4.1.2. Relativistic Proton Energy Distribution in the Jet. Under the assumptions discussed before, the system of transport equations (19) can be easily solved and the obtained proton distribution $N_{p}(E, z)$ is written as $[2,24]$

$$
N_{p}(E, z)=\int_{E_{p}}^{E_{p}^{\max }}\left|b_{p}(E)\right|^{-1} Q_{p}\left(E^{\prime}, z\right) e^{-\tau_{p}\left(E, E^{\prime}\right)} d E^{\prime}
$$

where

$$
\tau_{p}\left(E, E^{\prime}\right)=\int_{E}^{E^{\prime}} \frac{t_{\mathrm{esc}, p}^{-1} d E^{\prime \prime}}{\left|b_{p}\left(E^{\prime \prime}\right)\right|} .
$$

The quantity $Q_{p}(E, z)$ corresponds to the relativistic injection function of protons at the observer's frame (see the appendix). The minimum energy of protons is $E_{p}^{\mathrm{min}}=$ $E_{\text {th }}=1.22 \mathrm{GeV}$, while the maximum energy is assumed to be $E_{p}^{\max }=10^{6}-10^{7} \mathrm{GeV}$.

Figure 4 shows the proton energy distribution $N_{p}(E)$ at the base of the jet for M33 X-7 (solid line) and SS 433 (dashed line). As can be seen, the number of protons appears reduced (even by two orders of magnitude) for the wide halfopening angle system of M33 X-7 $\left(\xi=7^{\circ}\right)$ as compared to the narrow of SS $433\left(\xi=0.6^{\circ}\right)$. This is because the jet radius $r(z)$ is bigger and hence the magnetic field $B$ is smaller in the case of M33 X-7 than that of SS 433.

\subsection{Pion Injection Functions and Energy Distributions inside the Jet}

4.2.1. Pion Injection Functions. For pions produced through the inelastic p-p scattering, as injection function, we adopt the one given by [47] as

$$
Q_{\pi}(E, z)=n(z) c \int_{\varepsilon}^{1} \sigma_{p p}^{\text {inel }}\left(\frac{E}{x}\right) N_{p}\left(\frac{E}{x}, z\right) F_{\pi}\left(x, \frac{E}{x}\right) \frac{d x}{x},
$$

where $\varepsilon=E / E_{p}^{\max }, x=E / E_{p}$, and $F_{\pi}$ denotes the distribution of pions produced per $p-p$ collision (see the appendix).

4.2.2. Pion Energy Distributions. The steady-state energy distribution $N_{\pi}(E, z)$ for pions (created through the scattering of hot protons off the cold ones) results through a similar way to that of Equations (22) and (23). With the replacement $p \longrightarrow \pi$ and $t_{\mathrm{esc}}^{-1} \longrightarrow t_{\pi}^{-1}(E, z)$, respectively, on the latter equations, the corresponding solution for pion energy distribution is, then, written as

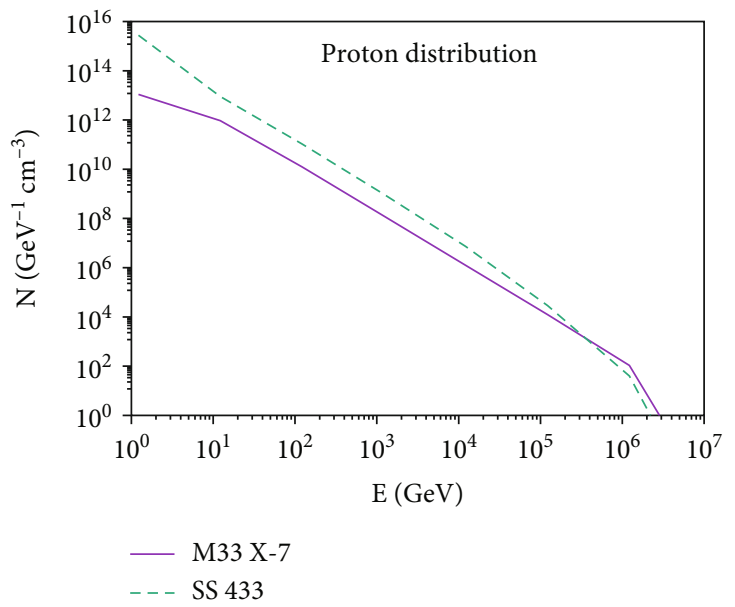

Figure 4: Proton energy distribution for M33 X-7 and SS 433 microquasar systems.

$$
N_{\pi}(E, z)=\int_{E}^{E_{\max }}\left|b_{\pi}(E)\right|^{-1} Q_{\pi}\left(E^{\prime}, z\right) e^{-\tau_{\pi}} d E^{\prime}
$$

where the rate $t_{\pi}^{-1}$ includes contributions from the decay and escape rates (see Equation (12)].

\subsection{Muon Injection Functions and Energy Distributions inside the Jet}

4.3.1. Muon Injection Functions. As mentioned before, in this work in addition to neutrinos coming from the decay of charged pions $\pi^{ \pm}$, we evaluate neutrino emissivities originating from the decay of the secondary charged muons $\mu^{ \pm}$. For the latter emissivity, we follow Reference [45], in order to take into account properly the muon energy loss. This means that it is necessary to consider the production of both left-handed and right-handed muons, $\mu_{L}^{-}$and $\mu_{R}^{+}$, separately, because $\mu_{L}^{-}$and $\mu_{R}^{+}$have different decay spectra.

Thus, the injection functions of the left-handed and right-handed muons are [45]

$$
\begin{aligned}
& Q_{\mu_{L}^{-}, \mu_{R}^{+}}\left(E_{\mu}, z\right)=\int_{E_{\mu}}^{E^{\max }} t_{\mathrm{dec}, \pi}^{-1}\left(E_{\pi}\right) N_{\pi}\left(E_{\pi}, z\right) \Theta\left(x-r_{\pi}\right) \times \frac{r_{\pi}(1-x)}{E_{\pi} x\left(1-r_{\pi}\right)^{2}} d E_{\pi}, \\
& Q_{\mu_{R}^{-}, \mu_{L}^{+}}\left(E_{\mu}, z\right)=\int_{E_{\mu}}^{E^{\max }} t_{\mathrm{dec}, \pi}^{-1}\left(E_{\pi}\right) N_{\pi}\left(E_{\pi}, z\right) \Theta\left(x-r_{\pi}\right) \times \frac{\left(x-r_{\pi}\right)}{E_{\pi} x\left(1-r_{\pi}\right)^{2}} d E_{\pi},
\end{aligned}
$$

with $x=E_{\mu} / E_{\pi}$ and $r_{\pi}=\left(m_{\mu} / m_{\pi}\right)^{2}$.

4.3.2. Muon Energy Distributions. The corresponding muon energy distributions, coming out of the transport equation, result from Equation (25) via the replacement $t_{\mathrm{esc}}^{-1} \longrightarrow t_{\mu}^{-1}(E, z)$, for muons, and are then written as 


$$
N_{\mu_{i}}(E, z)=\int_{E}^{E_{\max }}\left|b_{\mu}(E)\right|^{-1} Q_{\mu_{i}}\left(E^{\prime}, z\right) e^{-\tau_{\mu}} d E^{\prime}
$$

where the rate $t_{\mu}^{-1}$ for muons is given in Equation (12).

In Figure 5, the pion (a) and muon (b) energy distributions for M33 X-7 are illustrated. In both cases of particles, the distribution $N_{j}\left(E, z=z_{0}\right)$, with $j=\pi$ or $\mu$, which considers the most important energy losses, is compared with that which neglects energy losses, $N_{j, 0}\left(E, z=z_{0}\right)$. The solid lines correspond to distributions obtained by considering energy losses, and the dashed lines correspond to those where the energy losses obtained have been neglected.

\section{Results for Neutrino and $\gamma$-Ray Emissivities}

In this section, we present and discuss simulated emissivities of high-energy neutrinos and $\gamma$-rays produced in the extragalactic microquasar M33 X-7 system which is located in the Messier 33 galaxy, at a distance $\sim 840-960 \mathrm{kpc}$ from the Earth [33]. The derived algorithms for this purpose have been tested on the well-studied galactic microquasars SS 433 and Cyg X-1 system as well.

Because in our previous calculations on neutrino production from MQs [14-16] we neglected (due to the complexity and long time consuming) emissivity (intensity) of neutrinos originated from the secondary muons $\left(\mu^{ \pm}\right)$produced from the charged pion $\left(\pi^{ \pm}\right)$decays, in this section, we present contributions also originating from the $\mu^{ \pm}$channel (see Equation (5)).

By using the concentrations for protons $N_{p}$, pions $N_{\pi}$, and muons $N_{\mu}$, the neutrino and $\gamma$-ray intensities are subsequently calculated as described in the following.

5.1. Neutrino Emission from the p-p Reaction Family Tree. After the above discussion, the total emissivity $Q_{v}(E, z)$ produced from a MQ is the sum of contributions from the two sources: (i) the first comes from the direct $\pi^{ \pm}$decay (prompt neutrino production), and (ii) the second comes from the $\mu^{ \pm}$ decay (delayed neutrino production). Thus,

$$
Q_{v}(E, z)=Q_{\pi \longrightarrow v}(E, z)+Q_{\mu \longrightarrow v}(E, z)
$$

For pion decays, the injection function is given by

$$
Q_{\pi \longrightarrow v}(E, z)=\int_{E}^{E_{\max }} t_{\mathrm{dec}, \pi}^{-1}\left(E_{\pi}\right) N_{\pi}\left(E_{\pi}, z\right) \frac{\Theta\left(1-r_{\pi}-x\right)}{E_{\pi}\left(1-r_{\pi}\right)} d E_{\pi}
$$

with $x=E / E_{\pi}$, while for the four types of muon decays, the injection function reads

$$
\begin{aligned}
Q_{\mu \longrightarrow v}(E, z)= & \sum_{i=1}^{4} \int_{E}^{E_{\max }} t_{\mathrm{dec}, \mu}^{-1}\left(E_{\mu}\right) N_{\mu_{i}}\left(E_{\mu}, z\right) \\
& \times\left[\frac{5}{3}-3 x^{2}+\frac{4}{3} x^{3}+\left(3 x^{2}-\frac{1}{3}-\frac{8}{3} x^{3}\right) h_{i}\right] \frac{d E_{\mu}}{E_{\mu}},
\end{aligned}
$$

with $x=E / E_{\mu}$.

In the last expression, the symbols $\mu_{i}, i=1,2,3,4$ correspond to $\mu_{\{1,2\}}=\mu_{L}^{\{-,+\}}, \mu_{\{3,4\}}=\mu_{R}^{\{-,+\}}$[45], while $h_{\{1,2\}}=-h_{\{3,4\}}=-1$. Then, the calculation of each of the latter two integrals of Equations (29) and (30) provides separately the partial emissivity of neutrinos for the prompted and the delayed neutrino source, respectively. Needless to note that Earth and space telescope are not able to discriminate the two sources as it happens with laboratory neutrino sources.

Subsequently, we easily obtain the neutrino intensity (in units $\mathrm{GeV}^{-1} \mathrm{~s}^{-1}$ ) by the spatial integration

$$
I_{v}(E)=\int_{V} Q_{v}(E, z) d^{3} r=\pi(\tan \xi)^{2} \int_{z_{0}}^{z_{\max }} Q_{v}(E, z) z^{2} d z
$$

Figure 6(a) shows the neutrino intensity produced at the base of the jet from direct decays of secondary pions and muons coming from $\mathrm{p}-\mathrm{p}$ collisions in the jets of M33 X-7 and SS 433, respectively. As can be seen, the number of produced neutrinos reduces significantly for energies $E>10^{3}$ $\mathrm{GeV}$, following the behavior of pion and muon distribution. We can also see that the neutrinos produced in M33 X-7 are less than those produced in SS 433, due to the wider-half opening angle $\xi$ as we explained before.

It should be noted that, to perform the above calculations, we have updated and improved our codes to reduce the time consuming to a reasonable level, so as the integral involved in Equation (30) to be easily obtained, since in going the step from Equation (29) to Equation (30), the time consuming increases rapidly.

5.2. Gamma-Ray Emission from the p-p Reaction Chain. As we have discussed in Section 2, the p-p collisions inside the jets, produce secondary neutral particles (pions, eta particle, etc.) that decay to give $\gamma$-rays. The dominant of these channels goes through the reaction

$$
\pi^{0} \longrightarrow \gamma+\gamma
$$

For $E_{\gamma} \geq 100 \mathrm{GeV}$, we consider the $\gamma$-ray emissivity at a distance $z$ along the jets (in units $\mathrm{GeV}^{-1} \mathrm{~s}^{-1}$ ) to be given by [24] as

$$
Q_{\gamma}=c \int_{E_{\gamma} / E_{p}^{\max }}^{1} \sigma_{p p}^{\text {inel }}\left(\frac{E_{\gamma}}{x}\right) N_{p}\left(\frac{E_{\gamma}}{x}, z\right) F_{\gamma}\left(x, \frac{E_{\gamma}}{x}\right) \frac{d x}{x}
$$

where $F_{\gamma}$ is the spectrum of the produced $\gamma$-rays $[47,48]$ 


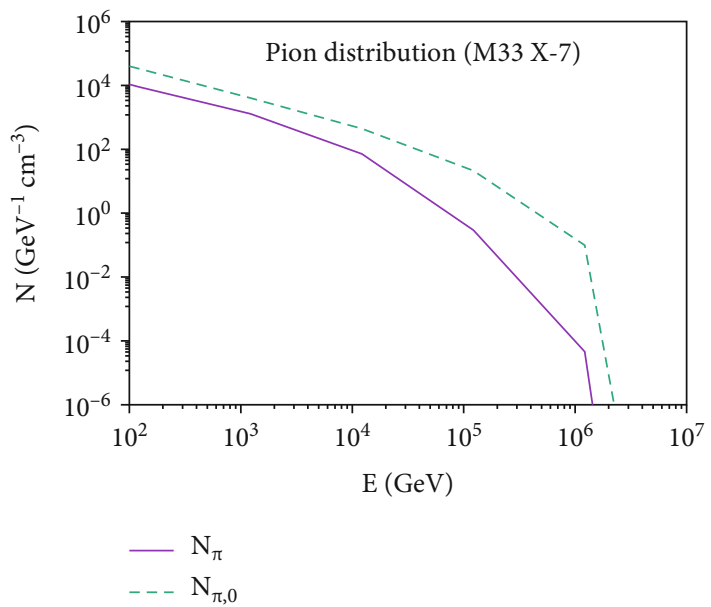

(a)

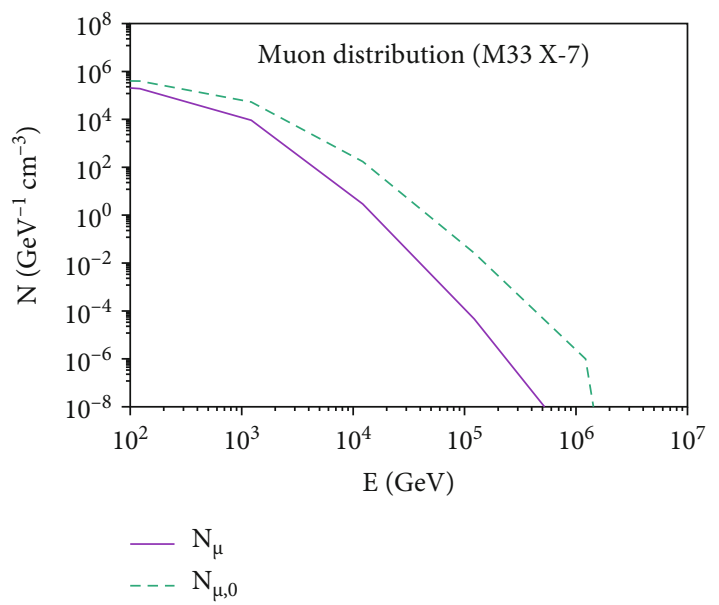

(b)

Figure 5: Pion (a) and muon (b) distributions for M33 X-7. In both cases of particles- $j$, we compare the distribution which considers the important energy losses $N_{j}\left(E, z=z_{0}\right)$ and with that which neglects energy losses $N_{j, 0}\left(E, z=z_{0}\right)$ for $j=\pi$, $\mu$. Both types of particle distributions refer to the base of the jet, i.e., at $z=z_{0}$.

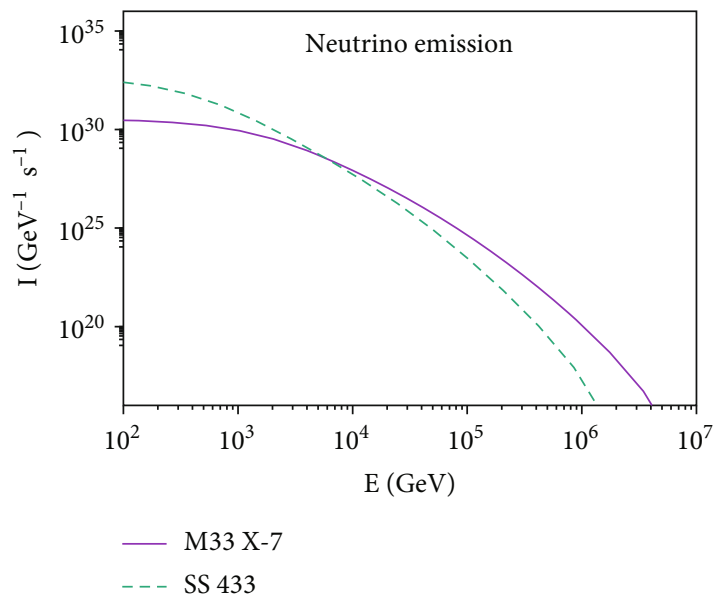

(a)

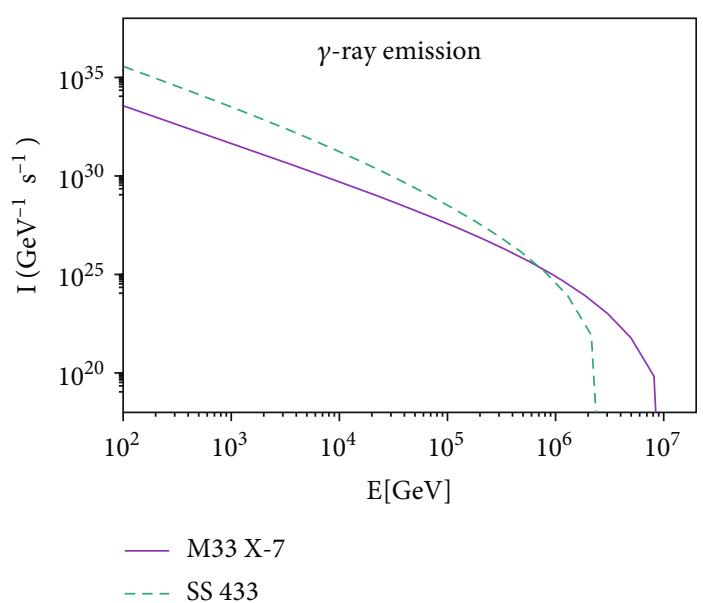

(b)

Figure 6: Neutrino (a) and gamma-ray (b) intensities for M33 X-7 and SS 433.

with energy $x=E_{\gamma} / E_{p}$ for a primary proton energy $E_{p}$. The function $F_{\gamma}$ is given in the appendix.

Subsequently, the corresponding spectral intensity of $\gamma$-rays can be obtained from the following spatial integration over the jet's volume $V$ as

$$
I_{\gamma}\left(E_{\gamma}\right)=\int_{V} Q_{\gamma}\left(E_{\gamma}, z\right) d^{3} r=\pi(\tan \xi)^{2} \int_{z_{0}}^{z_{\max }} Q_{\gamma}\left(E_{\gamma}, z\right) z^{2} d z .
$$

Figure 6(b) shows the $\gamma$-ray intensity for energies $E_{\gamma}>100$ $\mathrm{GeV}$. As can be seen, the produced $\gamma$-ray intensity reduces rather steadily following the behavior of the proton distribution in both cases of MQ systems. Again for the M33 X-7, it is lower mainly due to the wider half-opening angle (i.e., weaker magnetic field) but also due to other parameters.
5.3. Gamma-Ray and Neutrino Fluxes Arriving at the Earth. In the final stage of this work, we calculate neutrino and $\gamma$ -ray fluxes for the studied systems and compare them with existing observational data or sensitivities of the KM3NeT, IceCude, of the ANTARES detectors (for the neutrino fluxes), and of the CTA, MAGIC, and HESS telescopes (for the $\gamma$-ray fluxes). Towards this aim, we define the differential flux of neutrinos and $\gamma$-rays arriving at the Earth as [27]

$$
\frac{d \Phi}{d E}=\frac{1}{4 \pi d^{2}} I(E)
$$

where $d$ denotes the distance of the studied microquasar from Earth (see Table 1). Then, the differential fluxes weighted by the energy squared, $E^{2} d \Phi / d E$, can readily be obtained. 


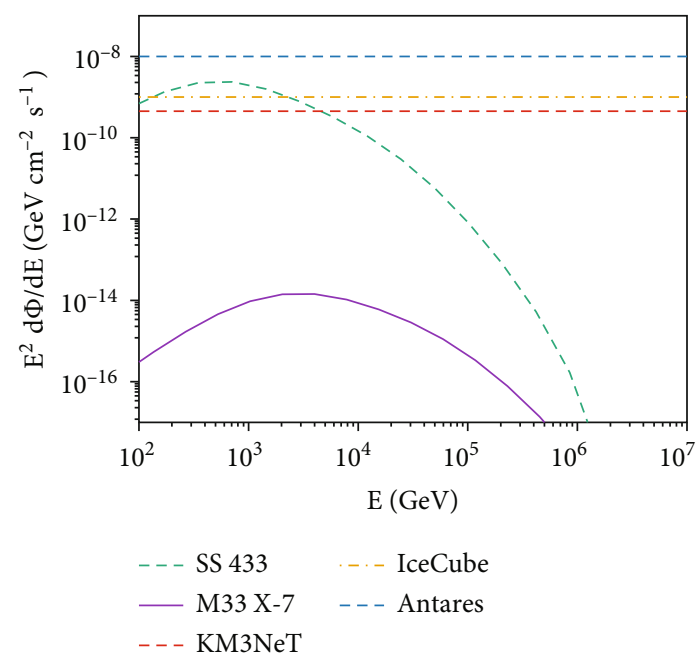

(a)

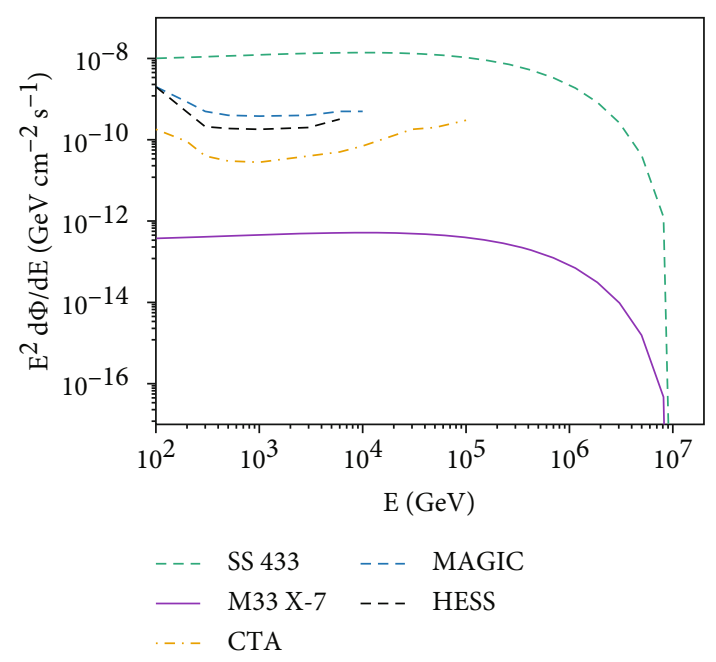

(b)

Figure 7: Differential neutrino fluxes (a) and gamma-ray fluxes (b) weighted over the energy squared $E^{2} d \Phi / d E$ for the M33 X-7 (solid lines) and SS 433 (dashed lines). They are compared with the corresponding sensitivities of the neutrino detectors (a) ANTARES [54], IceCube [55], and KM3NeT [56] and the respective gamma-ray sensitivities (b) from MAGIC and HESS telescopes as well as the foreseen sensitivity of the next-generation Cherenkov Telescope Array (CTA) [57].

Differential neutrino fluxes (left) and gamma-ray fluxes (right) weighted over the energy squared $E^{2} d \Phi / d E$ for the M33 X-7 (solid lines) and SS 433 (dashed lines). They are compared with the corresponding sensitivities of the neutrino detectors (left panel) ANTARES [54], IceCube [55], and $\mathrm{KM} 3 \mathrm{NeT}$ [56] and the respective gamma-ray sensitivities (right) from MAGIC and HESS telescopes as well as the foreseen sensitivity of the next-generation Cherenkov Telescope Array (CTA) [57].

In Figure 7, we illustrate the predictions of our model for the quantity $E^{2} d \Phi / d E$ produced at the base of the jets in the binary system M33 X-7 (solid lines), and the well-studied system SS 433 (dashed lines). In Figure 7(a), the comparison of these predictions for the neutrino fluxes with the corresponding sensitivities of the detectors ANTARES [54], IceCube [55], and $\mathrm{KM} 3 \mathrm{NeT}$ [56] is shown. Also, in Figure 7(b), the comparison of these predictions for the $\gamma$-ray fluxes with the corresponding sensitivities of the MAGIC and HESS telescopes, as well as the foreseen sensitivity of the next-generation Cherenkov Telescope Array (CTA) [57], is presented. In this subfigure, the observations are compared with our calculations obtained as described above.

As can be seen, our predictions are in good agreement with the available observations for SS 433 in the $\gamma$-ray energy range $10^{2}<E<10^{4}-10^{5} \mathrm{GeV}$. However, due to the much larger distance from Earth of the M33 X-1 system, relevant observations from present-day telescopes are not feasible. The corresponding $\gamma$-ray flux arriving at the Earth according to our calculations is about two orders of magnitude weaker than the sensitivity of the operating telescopes.

\section{Summary and Conclusion}

In this work, we address neutrino and $\gamma$-ray emissions from microquasars and X-ray binary stars (XRBs) that consist of a stellar-mass black hole (compact object) and a mainsequence donor star. We assume that these emissions originate from decay and scattering processes of the secondary particles produced through the p-p scattering mechanism, i.e., the inelastic collision of relativistic protons of the jet with the thermal ones.

Such high-energy neutrinos and $\gamma$-rays are detectable by operating terrestrial and space telescopes. Among the operating detectors are the under ice IceCube (at the South Pole), the ANTARES and KM3Net (under the Mediterranean sea), and many others.

In the near future, there are more accurate measurements of gamma-ray and neutrino fluxes by the coming observatories, such as the CTA (Acharya et al. 2018), which is expected to shed more light on the nature and the emission sources. From the perspective of neutrino detection, the addition of more years of data with continuous operation of IceCube will improve the sensitivity of the search for galactic sources of cosmic neutrinos. Furthermore, the next-generation detection instrument, IceCube-Gen2, a substantial expansion of IceCube, will be 10 times larger. This next-generation neutrino observatory with five times the effective area of IceCube is expected to improve the neutrino source search sensitivity by the same order [58]. With higher neutrino statistics, identifying galactic sources will become more promising.

On the other hand, theoretically, by modeling the solution of the system of coupled transport equations, we were able to perform detailed calculations for various processes taking place inside the jets of galactic and extragalactic system M33 X-7 assuming hadronic content in their jets. These cooling rates enter the proton, pion, and muon energy distributions through which one obtains neutrino and $\gamma$-ray intensities.

Our predictions for SS 433 in the $\gamma$-ray energy range 1 $0^{2}<E<10^{4}-10^{5} \mathrm{GeV}$ agree well with the observations. On the other hand, due to the much larger distance from Earth 
of the M33 X-1 MQ, relevant observations from present-day telescopes are not feasible since the corresponding fluxes arriving at the Earth are about two orders of magnitude weaker than the available sensitivities.

\section{Appendix}

\section{A. The Hot Proton's Injection Function}

At the observer's frame of reference, the injection function of protons, $Q_{p}(E, z)$, is given by [24]

$$
Q_{p}(E, z)=\left(\frac{z_{0}}{z}\right)^{3} \frac{Q_{0}}{\Gamma_{b}\left(E-\beta_{b} \cos \theta \sqrt{E^{2}-m^{2} c^{4}}\right)^{2}} \times\left[1-\frac{\beta_{b} E \cos \theta}{\sqrt{E^{2}-m^{2} c^{4}}}\right],
$$

where $\Gamma_{b}$ is the bulk Lorentz factor of the jet, and $\theta$ denotes the angle between the jet's injection axis and the direction of the line of sight (LOS).

Then, the normalization constant $Q_{0}$ is obtained by determining the power $L_{p}$ of the relativistic protons [24], given by

$$
L_{p}=\int_{V} d^{3} r \int_{E_{p}^{\min }}^{E_{p}^{\max }} E_{p} Q_{p}\left(E_{p}, z\right) d E_{p}
$$

After performing the latter integration, the normalization constant $Q_{0}$ (see text) reads [24]

$$
Q_{0}=\frac{2 c}{z_{0}} K_{0}, \quad K_{0}=\frac{4 q_{r e l} L_{k}}{c r_{0}^{2} \ln \left(E_{p^{\prime}}^{\max } / E_{p^{\prime}}^{\min }\right)}
$$

\section{B. The Distribution of Pions Produced per p-p Collision}

The distribution of pions produced per $\mathrm{p}-\mathrm{p}$ collision is

$$
\begin{aligned}
F_{\pi}^{(p p)}\left(x \cdot \frac{E}{x}\right)= & 4 \alpha B_{\pi} x^{\alpha-1}\left(\frac{1-x^{\alpha}}{1+r x^{\alpha}\left(1-x^{\alpha}\right)}\right)^{4} \\
& \times\left(\frac{1}{1-x^{\alpha}}+\frac{r\left(1-2 x^{\alpha}\right)}{1+r x^{\alpha}\left(1-x^{\alpha}\right)}\right)\left(1-\frac{m_{\pi} c^{2}}{x E_{p}}\right)^{1 / 2}
\end{aligned}
$$

with $x=E / E_{p}, \quad B_{\pi}=\alpha^{\prime}+0.25, \quad \alpha^{\prime}=3.67+0.83 L+0.075 L^{2}$, $r=2.6 / \sqrt{\alpha^{\prime}}$, and $\alpha=0.98 / \sqrt{\alpha^{\prime}}[46,47]$.

\section{Produced $\gamma$-Ray Spectrum}

Following the treatment of $[46,47]$, the spectrum of produced gamma-rays with energy $x=E_{\gamma} / E_{p}$ for a primary proton with energy $E_{p}$ is written as

$$
\begin{aligned}
F_{\gamma}\left(x, E_{p}\right)= & B_{\gamma} \frac{\ln x}{x}\left[\frac{1-x^{\beta_{\gamma}}}{1+k_{\gamma} x^{\beta_{\gamma}}\left(1-x^{\beta_{\gamma}}\right)}\right]^{4} \\
& \times\left[\frac{1}{\ln x}-\frac{4 \beta_{\gamma} x^{\beta_{\gamma}}}{1-x^{\beta_{\gamma}}}-\frac{4 k_{\gamma} \beta_{\gamma} x^{\beta_{\gamma}}\left(1-2 x^{\beta_{\gamma}}\right)}{1+k_{\gamma} x^{\beta_{\gamma}}\left(1-x^{\beta_{\gamma}}\right)}\right],
\end{aligned}
$$

where

$$
\begin{aligned}
& B_{\gamma}=1.3+0.14 L+0.011 L^{2}, \\
& \beta_{\gamma}=\frac{1}{0.008 L^{2}+0.11 L+1.79}, \\
& k_{\gamma}=\frac{1}{0.014 L^{2}+0.049 L+0.801},
\end{aligned}
$$

with

$$
L=\ln \left(\frac{E_{p}}{1 T e V}\right)
$$

\section{Data Availability}

There is no data used in this paper.

\section{Conflicts of Interest}

The authors declare that they have no conflicts of interest.

\section{Acknowledgments}

This research is cofinanced (O.T.K.) by Greece and the European Union (European Social Fund-ESF) through the Operational Programme "Human Resources Development, Education and Lifelong Learning 2014-2020" in the context of the project (MIS5047635). D.A.P. wishes to thank Prof. T.S. Kosmas for fruitful discussions during his stay in the Department of Physics, University of Ioannina.

\section{Supplementary Materials}

Supplementary 1. Gamma_Flux.

Supplementary 2. Neutrino_Flux.

\section{References}

[1] G. E. Romero, M. Boettcher, S. Markoff, and F. Tavecchio, "Relativistic jets in active galactic nuclei and microquasars," Space Science Reviews, vol. 207, p. 5, 2016.

[2] G. E. Romero and G. S. Vila, "The proton low-mass microquasar: high-energy emission.

[3] M. J. Reid et al., "The trigonometric parallax of Cygnus X-1," Astrophysical Journal, vol. 742, p. 83, 2011.

[4] T. Smponias and T. S. Kosmas, "Modelling the equatorial emission in a microquasar," Monthly Notices of the Royal Astronomical Society, vol. 412, pp. 1320-1330, 2011. 
[5] T. Smponias and T. S. Kosmas, "Dynamical and radiative simulations of $\gamma$-ray jets in microquasars," Monthly Notices of the Royal Astronomical Society, vol. 438, no. 2, pp. 1014-1026, 2014.

[6] F. L. Vieyro and G. E. Romero, "Particle transport in magnetized media around black holes and associated radiation," Astronomy and Astrophysics, vol. 542, no. A7, p. 1, 2012.

[7] M. G. Aartsen et al., "Search for prompt neutrino emission from gamma-ray bursts with IceCube collaboration," Astrophysical Journal Letters, vol. 805, p. L5, 2015.

[8] M. G. Aartsen et al., "Searches for sterile neutrinos with the IceCube detector (IceCube)," Physical Review Letters, vol. 117, no. 7, article 071801, 2016.

[9] M. G. Aartsen et al., "Neutrino emission from the direction of the blazar TXS $0506+056$ prior to the IceCube-170922A alert (IceCube)," Science, vol. 361, pp. 147-151, 2018.

[10] S. Adrián-Martínez, M. Ageron, F. Aharonian et al., "Letter of intent for KM3NeT 2.0," J. Phys. G: Nucl. Part. Phys., vol. 43, no. 8, article 084001, 2016.

[11] The CTA Consortium, M. Actis, G. Agnetta et al., "Design concepts for the Cherenkov telescope array CTA: an advanced facility for ground-based high-energy gamma-ray astronomy," Experimental Astronomy, vol. 32, no. 3, pp. 193-316, 2011.

[12] T. Y. Saito, R. Zanin, P. Bordas et al., "Microquasar observations with the MAGIC telescope," 2009, https://arxiv.org/abs/ 0907.1017v2.

[13] A. Albert, M. André, M. Anghinolfi et al., “ANTARES and IceCube combined search for neutrino point-like and extended sources in the southern sky," Astrophysical Journal, vol. 892, no. 2,2020 .

[14] T. Smponias and O. T. Kosmas, "High energy neutrino emission from astrophysical jets in the galaxy," Advances in High Energy Physics, vol. 2015, Article ID 921757, 7 pages, 2015.

[15] T. Smponias and O. Kosmas, "Neutrino emission from magnetized microquasar jets," Advances in High Energy Physics, vol. 2017, Article ID 4962741, 7 pages, 2017.

[16] O. T. Kosmas and T. Smponias, "Simulations of gamma-ray emission from magnetized microquasar jets," Advances in High Energy Physics, vol. 2018, Article ID 9602960, 11 pages, 2018.

[17] R. A. Remillard and J. E. McClintock, "X-ray properties of black-hole binaries," Annu Rev. Astron. Astrophys., vol. 44, no. 1, pp. 49-92, 2006.

[18] P. A. Charles and M. J. Coe, Compact Stellar X-Ray Sources, W. H. G. Lewin and M. Klis, Eds., Cambridge Univ. Press, Cambridge, UK, 2006.

[19] A. M. Cherepashchuk, K. A. Postnov, and A. A. Belinski, "Mass ratio in SS433 revisited," 2019, https://arxiv.org/abs/ 1902.11137.

[20] J. A. Orosz, "A massive star odyssey: from main sequence to supernova," in Proc. IAU Symp. 212, K. A. Hucht, A. Herrero, and C. Esteban, Eds., pp. 365-371, ASP, San Francisco, 2003.

[21] S. Fabrika, "The jets and supercritical accretion disk in SS433, Astrophys," Space Phys. Rev., vol. 12, p. 1, 2004.

[22] A. M. Cherepashchuk, R. A. Sunyaev, S. N. Fabrika et al., "SS 433: the accretion disk revealed in $\mathrm{H} \alpha$," Astronomy and Astrophysics, vol. 437, no. 2, pp. 561-573, 2005.

[23] I. F. Mirabel and L. F. Rodríguez, "Sources of relativistic jets in the galaxy," Annual Review of Astronomy and Astrophysics, vol. 37, pp. 409-443, 1999.
[24] M. M. Reynoso, G. E. Romero, and H. R. Christiansen, "Production of gamma rays and neutrinos in the dark jets of the microquasar SS433," Monthly Notices of the Royal Astronomical Society, vol. 387, pp. 1745-1754, 2008.

[25] D. A. Papadopoulos, "Study of gamma-ray and neutrino emission from microquasar jets," MSc Thesis, University of Ioannina (Unpublished), 2020.

[26] D. A. Papadopoulos, O. Kosmas, and S. Ganatsios, "Simulated emissions from astrophysical microquasar jets," to be submitted.

[27] M. M. Reynoso and G. E. Romero, "Magnetic field effects on neutrino production in microquasars," Astronomy and Astrophysics, vol. 493, pp. 1-11, 2009.

[28] I. G. Tsoulos, O. T. Kosmas, and V. N. Stavrou, "DiracSolver: a tool for solving the Dirac equation," Computer Physics Communications, vol. 236, p. 237, 2019.

[29] J. Albert, E. Aliu, H. Anderhub et al., "Very high energy gamma-ray radiation from the stellar mass black hole binary Cygnus X-1," Astrophysical Journal, vol. 665, no. 1, pp. L51L54, 2007.

[30] S. Sabatini, M. Tavani, P. Coppi et al., "Gamma-ray observations of Cygnus x-1 above $100 \mathrm{mev}$ in the hard and soft states," Astrophysical Journal, vol. 766, no. 2, p. 83, 2013.

[31] D. Kantzas, S. Markoff, T. Beuchert et al., "A new leptohadronic model applied to the first simultaneous multiwavelength data set for Cygnus X-1," Monthly Notices of the Royal Astronomical Society, vol. 500, no. 2, pp. 2112-2126, 2021.

[32] V. Bosch-Ramon et al., "The magnetic field and the location of the TeV emitter in Cygnus X-1 and LS 5039," Astronomy and Astrophysics, vol. 489, pp. L21-L24, 2008.

[33] W. Pietsch et al., "M33 X-7: ChASeM33 reveals the first eclipsing black hole X-ray binary," Astrophysical Journal, vol. 646, p. 420, 2006.

[34] J. D. Romney, R. T. Schilizzi, I. Fejes, and R. E. Spencer, "A series of VLBI images of SS433 during the outbursts in May/ June," Astrophysical Journal, vol. 321, p. 822, 1987.

[35] J. A. Orosz et al., "The mass of the black hole in Cygnus X-1," Astrophysical Journal, vol. 742, p. 84, 2011.

[36] D. R. Gies, C. T. Bolton, R. M. Blake et al., "Stellar wind variations during the X-ray high and low states of Cygnus X-11,2," Astrophysical Journal, vol. 678, no. 2, pp. 1237-1247, 2008.

[37] K. S. Long, S. Dodorico, P. A. Charles, and M. A. Dopita, "Observations of the X-ray sources in the nearby SC galaxy M33,” Astrophysical Journal, vol. 246, p. L61, 1981.

[38] F. Valsecchi, E. Glebbeek, W. M. Farr et al., "Formation of the black-hole binary M33 X-7 via mass-exchange in a tight massive system," 2010, https://arxiv.org/abs/1010.4809.

[39] J. A. Orosz, J. E. McClintock, R. Narayan et al., “A 15.65-solarmass black hole in an eclipsing binary in the nearby spiral galaxy M 33," Nature, vol. 449, pp. 872-875, 2007.

[40] A. Z. Bonanos K. Z. Stanek et al., "The first DIRECT distance determination to a detached eclipsing binary in M33," Astrophysical Journal, vol. 652, pp. 313-322, 2006.

[41] H. Falcke and P. Biermann, "The jet-disk symbiosis. I. Radio to $\mathrm{X}$-ray emission models for quasars," Astronomy and Astrophysics, vol. 293, p. 665, 1995.

[42] E. G. Körding, R. P. Fender, and S. Migliari, "Jet-dominated advective systems: radio and X-ray luminosity dependence on the accretion rate," Monthly Notices of the Royal Astronomical Society, vol. 369, p. 1451, 2006. 
[43] G. E. Romero, D. F. Torres, M. M. Kaufman Bernadó, and I. F. Mirabel, "Hadronic gamma-ray emission from windy microquasars," Astronomy and Astrophysics, vol. 410, pp. L1-L4, 2003.

[44] V. Bosch-Ramon, G. E. Romero, and J. M. Paredes, "A broadband leptonic model for gamma-ray emitting microquasars," Astronomy and Astrophysics, vol. 447, pp. 263-276, 2006.

[45] P. Lipari, M. Lusignoli, and D. Meloni, "Flavor composition and energy spectrum of astrophysical neutrinos," Phys. Rev. $D$, vol. 75, no. 12, article 123005, 2009.

[46] M. C. Begelman, S. P. Hatchett, C. F. McKee, C. L. Sarazin, and J. Arons, "Beam models for SS433," The Astrophysical Journal, vol. 238, pp. 722-730, 1980.

[47] S. R. Kelner, F. A. Aharonian, and V. V. Bugayov, "Energy spectra of gamma rays, electrons, and neutrinos produced at proton-proton interactions in the very high energy regime," Phys. Rev. D, vol. 74, article 034018, 2006.

[48] S. R. Kelner, F. A. Aharonian, and V. V. Bugayov, "Erratum: Energy spectra of gamma rays, electrons, and neutrinos produced at proton-proton interactions in the very high energy regime," Phys. Rev. D, vol. 79, no. 3, article 039901, 2009.

[49] T. K. Gaisser, Cosmic Rays and Particle Physics, Cambridge University Press, Cambridge, 1990.

[50] V. L. Ginzburg and S. I. Syrovatskii, The origin of cosmic rays, Pergamon Press Ltd., 1964.

[51] D. Khangulyan, S. Hnatic, F. Aharonian, and S. Bogovalov, "TeV light curve of PSR B1259-63/SS2883," Monthly Notices of the Royal Astronomical Society, vol. 380, no. 1, pp. 320330, 2007.

[52] O. T. Kosmas, 2020, in preparation.

[53] G. Ghisellini, L. Maraschi, and A. Treves, "Inhomogeneous synchrotron-self-Compton models and the problem of relativistic beaming of BL Lac objects," Astronomy and Astrophysics, vol. 146, p. 204, 1985.

[54] A. Albert, M. André, M. Anghinolfi et al., "First all-flavor neutrino pointlike source search with the antares neutrino telescope," Physical Review D, vol. 96, no. 8, article 082001, 2017.

[55] M. G. Aartsen et al., "(IceCube) all-sky search for timeintegrated neutrino emission from astrophysical sources with 7 yr of IceCube data," Astrophysical Journal, vol. 835, p. 151, 2017.

[56] S. Aiello et al., "Sensitivity of the KM3NeT/ARCA neutrino telescope to point-like neutrino sources," Astroparticle Physics, vol. 111, pp. 100-110, 2019.

[57] B. Sacco and S. Vercellone, "Present and future of the TeV astronomy with Cherenkov telescopes," 2010, https://arxiv .org/abs/1010.2208.

[58] M. G. Aartsen et al., "Neutrino astronomy with the next generation IceCube Neutrino Observatory (IceCube)," 2019, https:// arxiv.org/abs/1911.02561v1. 